\title{
Pore size distribution and supercritical hydrogen adsorption in activated carbon fibers
}

\author{
J J Purewal ${ }^{1}$, H Kabbour ${ }^{1,3}$, J J Vajo ${ }^{2}$, C C Ahn ${ }^{1}$ and B Fultz ${ }^{1}$ \\ ${ }^{1}$ Division of Engineering and Applied Science, California Institute of Technology, \\ Pasadena, CA, USA \\ ${ }^{2}$ HRL Laboratories, LLC, Malibu, CA, USA \\ E-mail: purewal@caltech.edu
}

Received 1 October 2008, in final form 25 November 2008

Published 23 April 2009

Online at stacks.iop.org/Nano/20/204012

\begin{abstract}
Pore size distributions (PSD) and supercritical $\mathrm{H}_{2}$ isotherms have been measured for two activated carbon fiber (ACF) samples. The surface area and the PSD both depend on the degree of activation to which the ACF has been exposed. The low-surface-area ACF has a narrow PSD centered at $0.5 \mathrm{~nm}$, while the high-surface-area ACF has a broad distribution of pore widths between 0.5 and $2 \mathrm{~nm}$. The $\mathrm{H}_{2}$ adsorption enthalpy in the zero-coverage limit depends on the relative abundance of the smallest pores relative to the larger pores. Measurements of the $\mathrm{H}_{2}$ isosteric adsorption enthalpy indicate the presence of energy heterogeneity in both ACF samples. Additional measurements on a microporous, coconut-derived activated carbon are presented for reference.
\end{abstract}

\section{Introduction}

One of the dilemmas of sorption-based hydrogen $\left(\mathrm{H}_{2}\right)$ storage in microporous carbons is the trade-off between adsorption capacity and adsorption enthalpy [1]. Conceptually, this problem is best illustrated in terms of the ideal slit pore formed from two parallel, semi-infinite graphite slabs. Packing density (and adsorption capacity) is maximized when the pore width is several times larger than the diameter of the $\mathrm{H}_{2}$ molecule, since it permits the formation of several hydrogen monolayers within the pore [2]. At supercritical temperatures (i.e. above $T_{\mathrm{c}}=33 \mathrm{~K}$ ) the $\mathrm{H}_{2}$-pore interactions are more dominant than the $\mathrm{H}_{2}-\mathrm{H}_{2}$ interactions and condensation within pores does not occur. Characteristic $\mathrm{H}_{2}$ adsorption enthalpies associated with large slit pores therefore approach the enthalpy of $\mathrm{H}_{2}$ adsorption on carbon, typically measured between 4 and $6 \mathrm{~kJ} \mathrm{~mol}^{-1}[3,4]$. With smaller pores, the adsorption enthalpy increases due to the overlap of potentials fields from each face of the pore. The packing density begins to decrease, though, when the pore is no longer wide enough to accommodate two $\mathrm{H}_{2}$ monolayers. Ab initio calculations of hydrogen adsorption in ideal slit pore carbons are largely

3 Present address: UCCS, Equipe Chimie du Solide, CNRS UMR 8181, ENSC Lille-UST Lille, BP 90108, 59652 Villeneuve d'Ascq cedex, France. consistent with this picture $[2,5]$. Experimental work on the fundamental limitations of hydrogen delivery from carbon sorbents is rare, however, because uniform slit pore structures do not occur in nature. To extend the slit pore concept to more realistic carbon adsorbents, the distribution of pore sizes must be understood.

Activated carbon fibers (ACFs), due to their microporosity, are an excellent material for a fundamental study of $\mathrm{H}_{2}$ adsorption capacity and enthalpy. Synthesized from polymeric carbon precursors, ACFs contain narrow and uniform pore size distributions with widths on the order of $1 \mathrm{~nm}[6,7]$. Images of ACFs from scanning tunneling microscopy have revealed networks of elongated slit-shaped and ellipsoidshaped pores $[8,9]$. Edge terminations in graphitic layers are thought to be the most reactive sites during the steam/carbon dioxide activation process, resulting in a gradual lengthening of slit-shaped pores as a function of burn-off [10]. ACFs subjected to less burn-off will have smaller pore volumes and a greater abundance of narrow pores widths. With longer activation times, the pore volume increases and the pores grow wider. This offers a convenient control for an experimental study of the correlation between pore structure and hydrogen adsorption.

In the current study, the pore size distribution (PSD) of activated carbon fibers is used to interpret the enthalpy and 
the capacity of supercritical $\mathrm{H}_{2}$ adsorption. Two ACF samples subjected to different degrees of activation are characterized. First, the microstructure of the activated carbon samples is investigated with high resolution transmission electron microscopy (HRTEM). Next, the PSD is obtained from argon adsorption measurements and modeling with the density functional theory (DFT) method. Supercritical hydrogen adsorption isotherms are then measured, from which the adsorption enthalpy and adsorption capacities are obtained. Similar measurements are made for a microporous, coconutderived activated carbon to determine whether self-consistent interpretations of hydrogen adsorption properties in terms of the PSD can be extended to other carbon sorbents.

\section{Experimental methods}

The Kynol ${ }^{\mathrm{TM}}$ activated carbon fiber samples ACF-1603-10 (ACF10) and ACF-1603-20 (ACF20) were obtained from Kynol Inc. These ACFs are produced by carbonization and steam/carbon dioxide gasification of a phenolic resin precursor. The last two digits in the labels are an approximate indication of surface area in hundreds of $\mathrm{m}^{2} \mathrm{~g}^{-1}$. The commercial, coconut-shell-derived activated carbon CNS-201 (CNS201) was purchased from A.C. Carbon Canada Inc. Because CNS201 is known to be highly microporous, containing $90 \%$ of slit pores smaller than $1.3 \mathrm{~nm}$, it is a useful reference material for the microporous ACFs [11]. Argon isotherms were measured at $87 \mathrm{~K}$ for ACF10 (0.2811 g) and ACF20 $(0.3222 \mathrm{~g})$. A nitrogen isotherm was measured at $77 \mathrm{~K}$ for CNS201 (0.1019 g). These measurements were made with a Micromeritics ASAP 2020. Surface areas and saturation pore volumes were calculated from the BET method and the Dubinin-Radushkevich (DR) method [12], respectively, as implemented in Micromeritics ASAP 2020 Version 3.01 software. Pore size distributions from the original DFT method were calculated using Micromeritics ASAP 2020 Version 3.01 software with DFT Plus ${ }^{4}$.

High resolution TEM micrographs were acquired on a Tecnai F30UT operated at $300 \mathrm{keV}$. Sample preparation consisted of grinding about $10 \mathrm{mg}$ of the ACF10 sample in isopropanol and dispersing it on a carbon grid.

High-pressure $\mathrm{H}_{2}$ isotherms were measured with a custom-built Sieverts apparatus at temperatures of 77, 87, and $195 \mathrm{~K}$. The Sieverts apparatus is equipped with a high resolution pressure manometer (MKS Baratron, Model 120AA-25000RBJ), and high vacuum molecular drag pump (Alcatel MDP 5011) with an oil-free diaphragm backing pump (KNF, Model N880.3 AN.22 E). The sample weights used for the hydrogen adsorption measurements were $0.1782 \mathrm{~g}$ (ACF10), $0.2114 \mathrm{~g}$ (ACF20) and $0.903 \mathrm{~g}$ (CNS201). Prior to adsorption measurements, samples were degassed by heating at $200{ }^{\circ} \mathrm{C}$ under vacuum for $12 \mathrm{~h}$. Sample masses were measured again after the adsorption experiment to check for changes in mass due to removal of residual water. The total volume of the Sieverts instrument, including both the reference volume and the reactor volume, is less than $68 \mathrm{~cm}^{3}$, permitting accurate

$4 \mathrm{Ar}$ and $\mathrm{N}_{2}$ isotherms, and DFT modeling performed by Micromeritics Analytical Services.

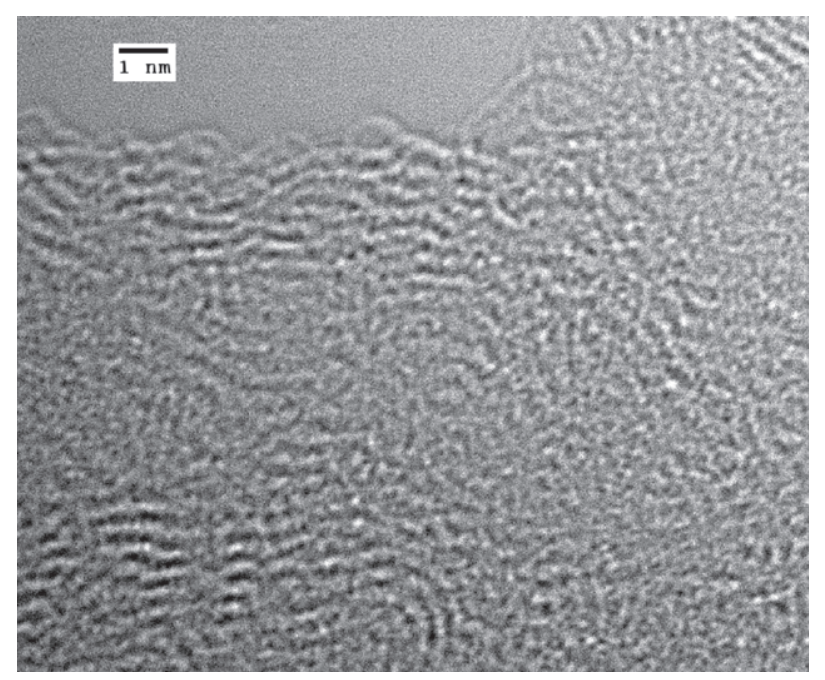

Figure 1. High resolution TEM image of ACF10. Length scale is indicated in the figure.

measurements using the volumetric method. The true sample volume was subtracted by assuming a skeletal density of $2.1 \mathrm{~g} \mathrm{ml}^{-1}$, which is known to be accurate for carbon samples. To account for real gas behavior, normal $\mathrm{H}_{2}$ gas densities were determined from measured temperatures and pressures using the RefProp Standard Reference Database [13].

\section{Results}

A high resolution TEM micrograph of ACF10 is presented in figure 1. Activated carbon fibers are known to be more graphitic than typical activated carbons [10], but this is not obvious from the HRTEM image. The microstructure appears to be random rather than showing well-ordered, regularly spaced graphitic planes. It appears similar to the highly disordered microstructures typically observed for activated carbon [14]. Micropore or mesopore structures were not visible with the HRTEM analysis. Since the HRTEM image is based on the exit electron wavefunction, however, the combination of finite sample thickness and non-periodicity tends to conceal micropore structures.

The BET surface areas and total pore volumes for ACF10, ACF20, and CNS201 are listed in table 1. As expected, ACF10 (subject to less burn-off than ACF20) has a smaller pore volume and smaller BET surface area than ACF20. The activated coconut carbon CNS201 appears to have a BET surface area and pore volume which is intermediate between the two ACFs (since this is the $\mathrm{N}_{2}$ BET surface area, direct comparisons with the Ar BET surface areas of ACF10 and ACF20 should be considered approximate). Calculated surface areas of activated carbons are often unrealistically high, since the micropore adsorption mechanism is not equivalent to the multilayer process assumed in the BET model. Nevertheless, the BET method is useful for comparisons between different materials.

Pore size distributions obtained from the DFT method are displayed in figure 2 . The pore width is defined as $L-d$, where 

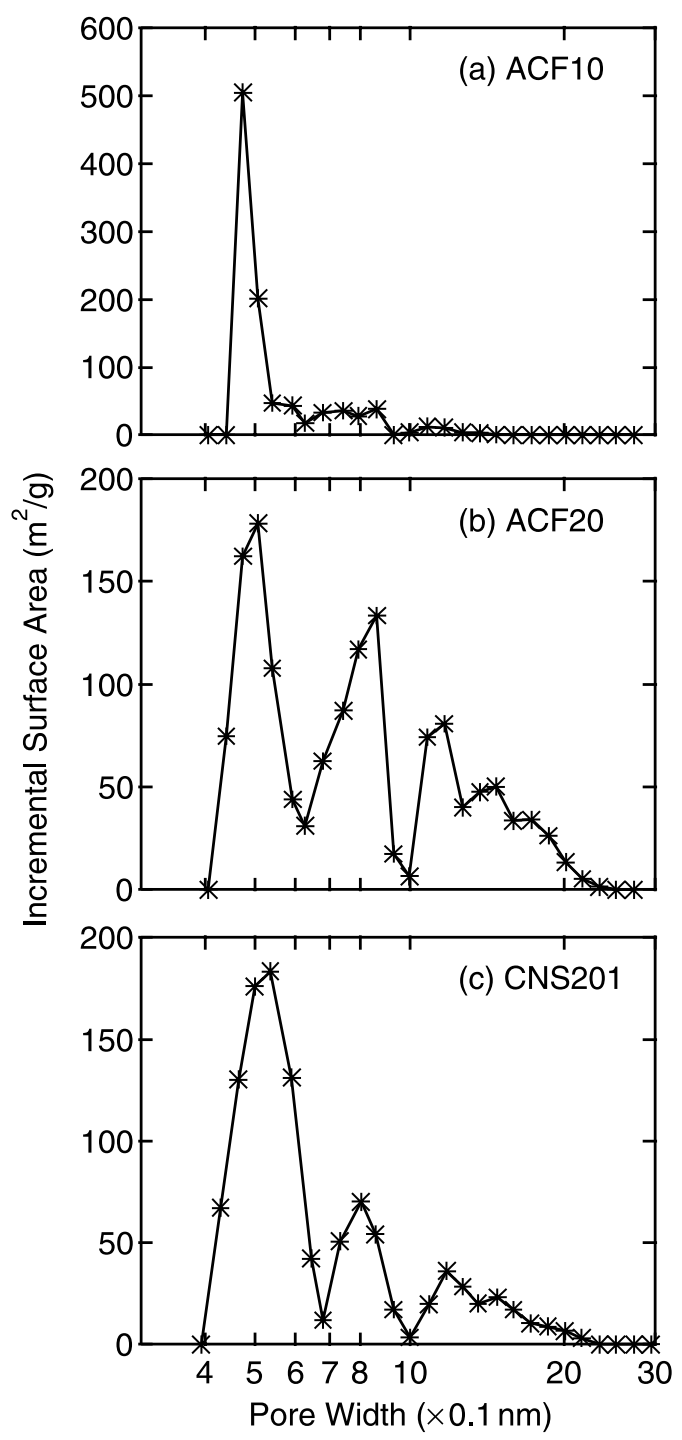

Figure 2. (a) Pore size distributions determined by the DFT method for (a) ACF10, (b) ACF20 and (c) CNS201. The bottom axis is shown in logarithmic scale.

Table 1. The BET specific surface areas (SSA) in $\mathrm{m}^{2} \mathrm{~g}^{-1}$ and total specific pore volumes (V) in $\mathrm{cm}^{3} \mathrm{~g}^{-1}$ from the

Dubinin-Radushkevich equation are listed. The textural parameters of ACF10 and ACF20 were obtained from argon measurements at $87 \mathrm{~K}$ while those of CNS201 were obtained from $\mathrm{N}_{2}$ measurements at $77 \mathrm{~K}$.

\begin{tabular}{lcl}
\hline & $\mathrm{SSA}\left(\mathrm{m}^{2} \mathrm{~g}^{-1}\right)$ & $\mathrm{V}\left(\mathrm{cm}^{3} \mathrm{~g}^{-1}\right)$ \\
\hline ACF1603-10 & 801 & 0.296 \\
ACF1603-20 & 1817 & 0.706 \\
CNS-201 & 1158 & 0.452 \\
\hline
\end{tabular}

$L$ is the internuclear spacing between the walls of the slit pore and $d$ is the van der Waals diameter of carbon $(0.34 \mathrm{~nm})$. Since graphite has an interlayer spacing of $L=0.335 \mathrm{~nm}$, a slit pore formed from a single missing graphene plane has a width of $0.33 \mathrm{~nm}$. A slit pore formed from two missing graphite planes has a width of $0.67 \mathrm{~nm}$, and a pore formed from three missing graphite planes has a width of $1.0 \mathrm{~nm}$. Taking into account the finite diameter of $\operatorname{Ar}(0.28 \mathrm{~nm})$ and $\mathrm{N}_{2}(0.30 \mathrm{~nm})$, the cutoff in the pore spectrum must be above $0.3 \mathrm{~nm}$. (Smaller probe molecules, such as helium could measure pore widths below this cutoff.)

The pore size distributions displayed in figure 2 all show a sharp increase in intensity just below $0.50 \mathrm{~nm}$, which corresponds to a width that is between the predicted pore widths for one or two removed graphite planes. This may simply reflect the smallest pore width which is measurable by the specific probe molecule. Another possibility is that after the removal of two layer planes, the slit pore structure relaxes to the observed $0.5 \mathrm{~nm}$ spacing. The pore distribution of ACF10 is narrow, consisting of the single large peak at $0.5 \mathrm{~nm}$. Since burn-off in ACF10 was minimized, larger pore structures did not develop fully, and are present only as a small tail in the PSD. In the pore size distribution of ACF20, there is a broad distribution of pore widths between 0.5 and $2.0 \mathrm{~nm}$ with a small positive skew. Since ACF20 was subject to a greater degree of burn-off, larger pores were formed during the activation process. The minima near 0.6 and $1.0 \mathrm{~nm}$ are likely to be model-induced artifacts which are independent of the actual sorbent material $[15]^{5}$. The coconut-derived activated carbon CNS201 also appears to have a distribution of pore widths between 0.5 and $2.0 \mathrm{~nm}$ with a positive skew. This broad distribution of pore widths in ACF20 and CNS201 may reflect the presence of slit pores formed from the removal of up to 6 graphite planes.

Supercritical $\mathrm{H}_{2}$ isotherms of ACF10, ACF20, and CNS201 are displayed in figure 3. Gibbs excess adsorption is the physical quantity that is measured experimentally. Therefore, supercritical isotherms often have a maximum at high pressures where the bulk gas density begins to increase faster than the adsorbate surface density [16, 17]. This behavior is visible in the isotherms of both ACF10 and ACF20. As shown in table 2, the $77 \mathrm{~K}$ adsorption capacities of ACF10, ACF20, and CNS201 are $2.1 \mathrm{wt} \%, 3.5 \mathrm{wt} \%$, and $2.5 \mathrm{wt} \%$, respectively. Comprehensive studies of $\mathrm{H}_{2}$ adsorption in activated carbons report a linear dependence of storage capacity at $77 \mathrm{~K}$ with specific surface area [18-21]. When the $\mathrm{H}_{2}$ adsorption capacity is expressed as a mass\%, an increase of approximately $1 \mathrm{wt} \%$ hydrogen is observed for every $500 \mathrm{~m}^{2} \mathrm{~g}^{-1}$ of surface area. As shown in the inset of figure 3 , the $\mathrm{H}_{2}$ adsorption capacity of ACF10, ACF20, and CNS201 at $77 \mathrm{~K}$ roughly follow this linear relation.

At low surface excess concentration, Henry's law gives a linear relationship between the amount of adsorption and the equilibrium pressure, $n=k_{\mathrm{H}} \times p$. Adsorption in the 'Henry's law' region can be even more accurately modeled by a virial-type equation to take into account small deviations from linearity [6],

$$
\ln (n / p)=C_{1}+C_{2} n+C_{3} n^{2}+\cdots .
$$

5 The minima in the distributions have been proposed to originate from packing effects where the pore width transitions from being able to support one adsorbed layer to two and from two layers to three. This width is governed by the functional form used for the adsorbing molecule, for example, a LennardJones potential. However, the fact that the maxima in the distributions are separated by integrals of the graphite layer spacing distance suggests that the maxima may be real and correspond to pores formed from removal of one, two, three, etc, graphitic planes. 


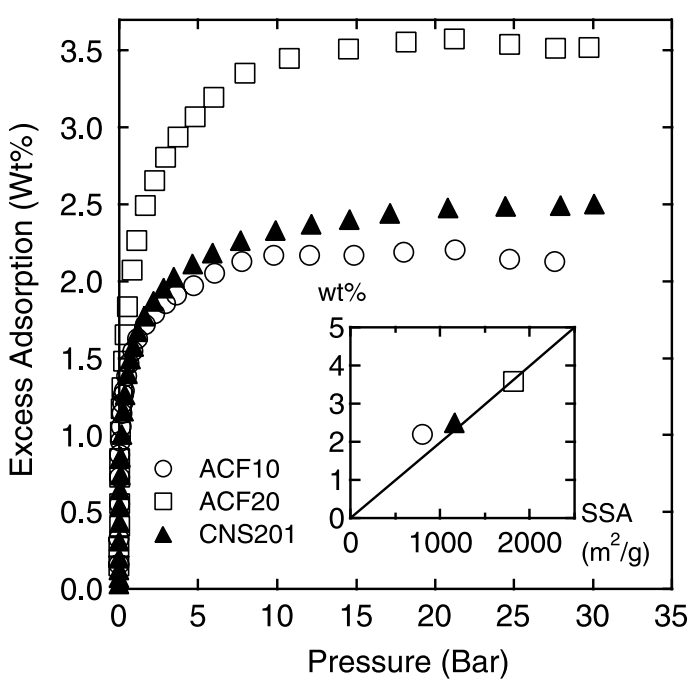

Figure 3. Hydrogen adsorption isotherms at $77 \mathrm{~K}$ of (open circle) ACF10, (open square) ACF20 and (solid triangle) CNS201. Inset: excess gravimetric density $\left(\mathrm{H}_{2} \mathrm{wt} \%\right)$ saturation value at $77 \mathrm{~K}$ as a function of BET specific surface area $\left(\mathrm{m}^{2} \mathrm{~g}^{-1}\right)$, with the solid line signifying a $1 \mathrm{wt} \%$ per $500 \mathrm{~m}^{2} \mathrm{~g}^{-1}$ linear relationship.

Table 2. Adsorption enthalpy at zero coverage and saturation $\mathrm{H}_{2}$ adsorption amounts at $77 \mathrm{~K}$. For clarity, positive enthalpies are displayed even though adsorption is exothermic.

\begin{tabular}{lll}
\hline Material & $\Delta H_{0}\left(\mathrm{~kJ} \mathrm{~mol}^{-1}\right)$ & $\mathrm{H}_{2}(\mathrm{wt} \%)$ \\
\hline ACF10 & 8.92 & 2.1 \\
ACF20 & 7.63 & 3.5 \\
CNS201 & 8.59 & 2.5 \\
\hline
\end{tabular}

Accurate adsorption amounts were measured in the lowpressure region using a high resolution pressure gauge. Henry's law constants were then calculated at 77, 87 and $195 \mathrm{~K}$ from the zero-order virial coefficient, $C_{1}=\ln \left(k_{\mathrm{H}}\right)$. The differential enthalpy of adsorption was calculated from the temperature variation of $k_{\mathrm{H}}$ calculated using the van't Hoff expression,

$$
\Delta H_{0}=R\left(\frac{\partial \ln k_{\mathrm{H}}}{\partial 1 / T}\right)_{n} .
$$

In all the samples $\ln \left(k_{\mathrm{H}}\right)$ varies linearly with $1 / T$, and an accurate estimate of $\Delta H_{0}$ is obtained from the slope. Values of enthalpy in the zero-coverage limit are summarized in table 2. For clarity, the absolute values of $\Delta H_{0}$ are displayed. The largest adsorption enthalpy $\left(8.92 \mathrm{~kJ} \mathrm{~mol}^{-1}\right)$ is observed for ACF10, which has the largest fraction of narrow pores. In ACF20, which has larger pore structures, the adsorption enthalpy $\left(7.63 \mathrm{~kJ} \mathrm{~mol}^{-1}\right)$ is smaller. An intermediate enthalpy is observed for the activated carbon CNS201.

The isosteric heat is defined as the absolute value of the enthalpy change of the system upon the adsorption of an infinitesimal surface excess amount [6]. It is calculated from the 77 and $87 \mathrm{~K}$ isotherms using the following thermodynamic expression,

$$
\Delta H=R\left(\frac{\partial \ln p}{\partial 1 / T}\right)_{n}
$$

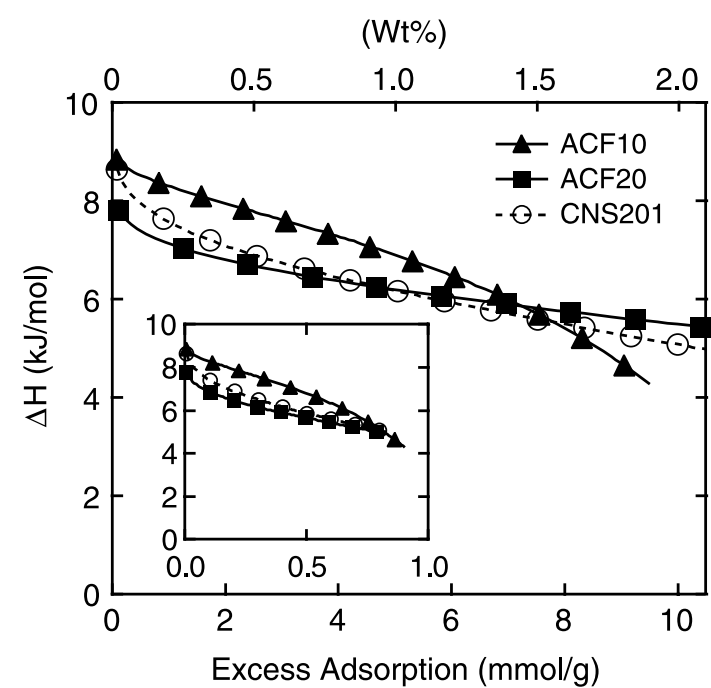

Figure 4. The $\mathrm{H}_{2}$ isosteric enthalpy of adsorption for ACF10, ACF20 and CNS201 as a function of excess adsorption. Inset: the isosteric heat is plotted as a function of the fractional amount of adsorption (excess adsorption divided by the saturation adsorption amount at $77 \mathrm{~K})$.

As displayed in figure 4, all of the samples are energetically heterogeneous, since the enthalpy decreases with increasing adsorption amount. The limiting values of $\Delta H$ at zero coverage compare favorably with the values in table 2 obtained from the virial expansion. Heterogeneity is especially pronounced for ACF10, which decreases from $8.3 \mathrm{~kJ} \mathrm{~mol}^{-1}$ at $1 \mathrm{mmol} \mathrm{g}^{-1}$ to $5.4 \mathrm{~kJ} \mathrm{~mol}^{-1}$ at $8 \mathrm{mmol} \mathrm{g}^{-1}$. Since the isosteric heats are not homogeneous, it is useful to take an average. The average isosteric heat between 0 and $9.5 \mathrm{mmol} \mathrm{g}^{-1}$ is $6.64 \mathrm{~kJ} \mathrm{~mol}^{-1}$ for ACF10 and $5.85 \mathrm{~kJ} \mathrm{~mol}^{-1}$ for ACF20. For CNS201 the average is $6.07 \mathrm{~kJ} \mathrm{~mol}^{-1}$. In the inset to figure 4 , the isosteric heat is also plotted against the excess adsorption expressed as a fraction of the adsorption capacity at $77 \mathrm{~K}$. When viewed as a function of fractional adsorption, the isosteric heat of ACF10 remains higher than ACF20 until a fractional adsorption of about 0.8 . It should be noted that the isosteric heats may not be accurate at high fractional fillings, however, due to the nearly flat plateaus of the isotherms in that region.

\section{Discussion}

Heterogeneity in the adsorption potentials of microporous carbons is modeled using a distribution of widths of the slit pores. Recent studies have also stressed the importance of fluctuations in pore wall thickness on the adsorption potential $[22,1]$. Regardless of wall thickness, though, narrow pores tend to have deeper potential wells. Therefore, in terms of the Polanyi adsorption potential

$$
A=-R T \ln \left(p / p_{0}\right),
$$

the smallest pores are filled at the lowest pressures, followed by the gradual filling of larger pores at higher pressures. At a given equilibrium pressure, $p$, there should be a critical 
pore width, below which all pores are filled and above which all pores are vacant [23]. The zero-coverage heat of $\mathrm{H}_{2}$ adsorption, $\Delta H_{0}$, reflects the initial filling of pores, and therefore it should depend on the PSD of the three samples.

Since ACF10 contains a narrow peak at $0.5 \mathrm{~nm}$ in the PSD, the filling of these small pores leads to the large zerocoverage $\mathrm{H}_{2}$ adsorption enthalpy. ACF10 also contains a small fraction of wider pores, and it is the filling of these larger pores that apparently causes the steep decrease in the isosteric heat of adsorption at higher coverage. In ACF20 there is a broad distribution of pore widths. In theory, the smallest $0.5 \mathrm{~nm}$ pores should fill first, leading to a similar zero-coverage $\mathrm{H}_{2}$ adsorption enthalpy as ACF10. Measurements indicate, however, that ACF20 has a significantly smaller zero-coverage enthalpy than ACF10. The exclusive filling of the smallest pores at low pressures, in isolation from the larger pores, may not be a realistic view. Simultaneous filling of pores with widths between 0.5 and $2.0 \mathrm{~nm}$, on the other hand, might explain this smaller-than-expected zero-coverage adsorption enthalpy of ACF20. It is the relative abundance of the smallest pores relative to the larger pores which seems to have the greatest influence on the zero-coverage enthalpy.

In all three samples, the isosteric heat of adsorption decreases with the excess adsorption amount. Since the samples have different adsorption capacities, a better comparison can be made by plotting the isosteric heats as a function of fractional adsorption. This is displayed in the inset to figure 4. The isosteric heats of both ACF10 and ACF20 decrease almost uniformly at low filling, but ACF10 eventually falls below ACF20 at a fractional filling of 0.8. At least some of the decrease in isosteric heat can be attributed to interactions between the sorbed $\mathrm{H}_{2}$ molecules. These unfavorable interactions are likely to be greater in small slit pores than in larger slit pores, leading to the observed behavior.

Heats of adsorption in the Henry's Law region fall between 7.63 and $8.92 \mathrm{~kJ} \mathrm{~mol}^{-1}$ in table 2, greater than the 4-6 kJ mol ${ }^{-1}$ energies typically observed for $\mathrm{H}_{2}$ adsorption on carbon. This is due to strong interactions between sorbed $\mathrm{H}_{2}$ molecules and the slit pore potential fields. Average isosteric heats of adsorption are between 5.8 and $6.7 \mathrm{~kJ} \mathrm{~mol}^{-1}$, closer to the characteristic values for carbon. For the three samples studied, the $\mathrm{H}_{2}$ adsorption enthalpy was lower for the samples having higher capacity.

\section{Conclusion}

Pore size distributions and supercritical $\mathrm{H}_{2}$ isotherms for two microporous activated carbon fibers and one coconut-derived activated carbon were measured and compared. The low surface area sample, ACF10, has a narrow peak in the PSD at $0.50 \mathrm{~nm}$ with a very small tail at larger widths. The high surface area sample, ACF20, has a broad PSD which contains widths between 0.5 and $2.0 \mathrm{~nm}$ and has a small positive skew. The coconut-derived activated carbon CNS201 also has a broad PSD with widths between 0.5 and $2.0 \mathrm{~nm}$. The zero-coverage enthalpy of $\mathrm{H}_{2}$ adsorption depends strongly on the relative intensity of the smallest pore widths in the PSD. Zero-coverage enthalpies vary from $8.92 \mathrm{~kJ} \mathrm{~mol}^{-1}$ for low surface area ACF10 to $7.63 \mathrm{~kJ} \mathrm{~mol}^{-1}$ for high surface area ACF20. The variation of the isosteric enthalpies with excess adsorption suggests the presence of unfavorable $\mathrm{H}_{2}-\mathrm{H}_{2}$ interactions within slit pores in addition to the presence of energetically heterogeneous sorption sites. The $\mathrm{H}_{2}$ adsorption enthalpy was lower for the samples having higher $\mathrm{H}_{2}$ adsorption capacity.

\section{Acknowledgments}

We thank A Dailly for useful discussions. Work was funded by the DOE Office of Energy Efficiency and Renewable Energy through DE-FC36-05GO15079. Collaboration for this work was through the Center of Excellence on Carbon-Based Hydrogen Storage Materials.

\section{References}

[1] Bhatia S K and Myers A L 2006 Optimum conditions for adsorptive storage Langmuir 221688

[2] Wang Q and Johnson J K 1999 Molecular simulation of hydrogen adsorption in single-walled carbon nanotubes and idealized carbon slit pores J. Chem. Phys. 110577

[3] Cheng H S, Cooper A C, Pez G P, Kostov M K, Piotrowski P and Stuart S J 2005 Molecular dynamics simulations on the effects of diameter and chirality on hydrogen adsorption in single-walled carbon nanotubes J. Phys. Chem. B 1093780

[4] Pace E L and Siebert A R 1959 Heat of adsorption of parahydrogen and orthodeuterium on graphon J. Phys. Chem. 63 1398-400

[5] Gigras A, Bhatia S K, Anil Kumar A V and Myers A L 2007 Feasibility of tailoring for high isosteric heat to improve effectiveness of hydrogen storage in carbons Carbon 451043

[6] Rouquerol F, Rouquerol J and Sing K 1999 Adsorption by Powders and Porous Solids (London: Academic)

[7] Stoeckli F, Centeno T A, Fuertes A B and Muniz J 1996 Porous structure of polyarylamide-based activated carbon fibres Carbon 341201

[8] Daley M A, Tandon D, Economy J and Hippo E J 1996 Elucidating the porous structure of activated carbon fibers using direct and indirect methods Carbon 34 1191-1200

[9] Paredes J I, Martínez-Alonso A and Tascón J M D 2001 Characterization of microporosity and mesoporosity in carbonaceous materials by scanning tunneling microscopy Langmuir 17474

[10] Yang R T 2003 Adsorbents: Fundamental and Applications (Hoboken, New Jersey: Wiley Interscience)

[11] Bénard P and Chahine R 1997 Modeling of high-pressure adsorption isotherms above the critical temperature on microporous adsorbents: application to methane Langmuir 13808

[12] Dubinin M M 1967 J. Colloid Interface Sci. 23487

[13] Lemmon E W, Huber M L and McLinden M O 2007 NIST Standard Reference Database 23: Reference Fluid Thermodynamic and Transport Properties-REFPROP Number Version 8.0 in Standard Reference Data Program. National Institute of Standards and Technology, Gaithersburg

[14] Baker F S, Miller C E, Repik A J and Tolles E D 2003 Activated carbon Kirk-Othmer Encyclopedia of Chemical Technology vol 4 (New York: Wiley) p 741

[15] Olivier J P 1998 Improving the models used for calculating the size distribution of micropore volume of activated carbons from adsorption data Carbon 361469 
[16] Bénard P and Chahine R 2001 Determination of the adsorption isotherms of hydrogen on activated carbons above the critical temperature of the adsorbate over wide temperature and pressure ranges Langmuir 17 1950-5

[17] Dillon A C and Heben M J 2001 Hydrogen storage using carbon adsorbents: past, present and future Appl. Phys. A 72133

[18] Chahine R and Bose T K 1996 11th WHEC (Oxford: Pergamon) p 1259

[19] Kabbour H, Baumann T F, Satcher J H Jr, Saulnier A and Ahn C C 2006 Toward new candidates for hydrogen storage: high-surface-area carbon aerogels Chem. Mater. 186085
[20] Panella B, Hirscher M and Roth S 2005 Hydrogen adsorption in different carbon nanostructures Carbon 43 2209-14

[21] Nijkamp M G, Raaymakers J E M J, van Dillen A J and de Jong K P 2001 Hydrogen storage using physisorption-materials demands Appl. Phys. A 72 619-23

[22] Nguyen T X and Bhatia S K 2005 Characterization of activated carbon fibers using argon adsorption Carbon $\mathbf{4 3} 775$

[23] Zhou L 2002 Adsorption isotherms for the supercritical region Adsorption: Theory, Modeling, and Analysis (Surfactant Science Series vol 107) ed J Tóth (New York: Dekker) p 211 